\title{
Association between Dietary Patterns and Non-alcoholic Fatty Liver Disease: Results from a Case-Control Study
}

\author{
Helda Tutunchi, PhD; Maryam Saghafi-Asl, PhD²; Mohammad Asghari-Jafarabadi, PhD; Alireza Ostadrahimi, MD, PhD²* \\ ${ }^{1}$ Student Research Committee, Nutrition Research Center, Department of Clinical Nutrition, School of Nutrition \& Food Sciences, Tabriz \\ University of Medical Sciences, Tabriz, Iran \\ ${ }^{2}$ Nutrition Research Center, Department of Clinical Nutrition, School of Nutrition \& Food Sciences, Tabriz University of Medical Sciences, \\ Tabriz, Iran \\ ${ }^{3}$ Road Traffic Injury Research Center, Tabriz University of Medical Sciences, Tabriz, Iran
}

\begin{abstract}
Background: This study aimed to investigate the association between dietary patterns and non-alcoholic fatty liver disease (NAFLD) among Iranian adults.

Methods: This case-control study was conducted on 210 subjects. NAFLD diagnosis was made by ultrasound examination. Anthropometric measures, physical activity, fasting serum levels of glucose, alanine aminotransferase (ALT), aspartate aminotransferase, and lipid profile were assessed. A three-day food diary was used to assess dietary intakes of the subjects. Dietary patterns were determined using factor analysis. To determine the relationship between dietary patterns and NAFLD, multivariableadjusted odds ratio (OR) obtained from the logistic regression analysis was used.

Results: Two dietary patterns were extracted as follows: vegetables, legumes, fruits, and low-fat dairy products (VLFD) ; and sweet, hydrogenated fat, red and processed meat, and soft drink (SHMS) dietary patterns. By taking all possible confounders into account, the VLFD dietary pattern was found to be significantly related to lower odds of NAFLD, while the SHMS dietary pattern was independently related to higher odds of NAFLD $(P<0.05)$. Among major food groups, high consumption of processed meat, hydrogenated fats, sweets and desserts, and soft drinks was positively related to NAFLD $(P<0.05)$, whereas vegetable consumption exhibited a protective role against NAFLD $(P=0.01)$.

Conclusion: The VLFD dietary pattern was associated with reduced likelihood of having NAFLD, while the SHMS dietary pattern was associated with higher likelihood. Therefore, the VLFD dietary pattern might be useful in the nutritional strategies for NAFLD patients. Further studies with larger sample sizes and prospective design are warranted.

Keywords: Adult population, Dietary patterns, Factor analysis, Non-alcoholic fatty liver (NAFLD)

Cite this article as: Tutunchi H, Saghafi-Asl M, Asghari-Jafarabadi M, Ostadrahimi A. Association between dietary patterns and non-alcoholic fatty liver disease: results from a case-control study. Arch Iran Med. 2021;24(1):35-42. doi: 10.34172/aim.2021.06.
\end{abstract}

Received: February 29, 2020, Accepted: October 27, 2020, ePublished: January 1, 2021

\section{Introduction}

With the growing prevalence of obesity worldwide, much attention has been given to the metabolic syndrome as well as its related hepatic manifestation, non-alcoholic fatty liver disease (NAFLD). ${ }^{1,2}$ This disease is defined as a liver pathologic spectrum initiated by simple hepatic steatosis that can progress to steatohepatitis, fibrosis, cirrhosis, and finally to liver cancer. ${ }^{1}$ Although the real prevalence of NAFLD may vary depending on the sensitivity of the detection method used, recently, there has been a significant increase in the prevalence of NAFLD. To date, the prevalence rate of NAFLD has been estimated between $8 \%$ and $45 \%$ worldwide. ${ }^{3}$ However, NAFLD prevalence is estimated to range from $42 \%$ to $93 \%$ in obese subjects and in patients with the metabolic syndrome., ${ }^{3,4}$ The prevalence of NAFLD has also increased recently in Asian countries due to the rise of overweight/obesity, diabetes, and the metabolic syndrome among the population in this region., ${ }^{5,6}$ Lifestyle modifications including a healthy diet, weight reduction, and increasing physical activity remain the first-line therapies for NAFLD. ${ }^{7-12}$ Western lifestyle characterized by decreased physical activity and consumption of diets that are rich in animal fat, saturated fats, processed meat, red meat, refined grains, and sweets is associated with increased risk of NAFLD. ${ }^{13,14}$

Most of the previous studies assessing the association between diet and NAFLD have focused on the traditional dietary assessment procedures, and investigated the effect of separate nutrients or foods. This method has shown conflicting findings difficult to be interpreted due to problems of isolating the impacts of specific nutrients or foods on the outcome. ${ }^{15}$ Rather than looking at individual nutrients, a dietary pattern analysis evaluates the effects of overall diet, including interactions and synergistic effects among nutrients and foods. ${ }^{16}$ Therefore, dietary patterns may be more informative for assessing the diet-disease associations compared to individual foods or nutrients. ${ }^{17}$ In recent studies, much attention has been paid to 
the association between overall dietary patterns and NAFLD. ${ }^{18-20}$ Correspondingly, it was demonstrated that a Mediterranean dietary pattern is inversely correlated with NAFLD. ${ }^{8,21,22}$ In contrast, an unhealthy dietary pattern has been shown to increase the risk of NAFLD as well as its risk factors. ${ }^{8}$ However, to the best of our knowledge, there is limited research performed on the association between overall dietary patterns and NAFLD among Iranian adults. Therefore, the current study was designed to examine the relationship between dietary patterns and NAFLD in a sample of Iranian adults using a matched case-control design.

\section{Materials and Methods}

Study Population

This matched case-control study was carried out between May and November 2019 in northwestern Iran. Patients were recruited from the gastrointestinal clinic at Imam Reza hospital in northwestern Iran. The present study included 105 subjects newly diagnosed with NAFLD. After recruitment of all cases, 105 healthy individuals without NAFLD (from the same clinic) were recruited as the control group. Each patient was matched to a control of the same sex and age. The sample size of the study was calculated based on the information from the main outcomes of the study, including weight, body mass index (BMI), waist circumference (WC) and alanine aminotransferase (ALT). Considering a confidence of $95 \%$, a power of 95 , the sample size was estimated by the PASS 15 software to be 66, 67, 101 and 21 per group, respectively for weight, BMI, WC and ALT. ${ }^{23,34}$ To cover the sample size required for all risk factors of the study, we chose the maximum sample size estimated. The primary information to calculate sample size was obtained from a study by Soleimani et $\mathrm{al}^{18}$ wherein the information on the main outcome corresponding to the maximum sample size was: WC $(\mathrm{cm})$ : cases (mean $\pm \mathrm{SD}): 102.3 \pm 13.5$, controls (mean \pm SD): $96.3 \pm 15.9$.

The inclusion criteria for the case and control groups were subjects aged 30 to 60 years, and those with ultrasounddiagnosed NAFLD for the cases and without NAFLD as detected by ultrasound for the controls. Patients with liver diseases such as hepatitis and biliary disease and inherited disorders affecting liver, those with kidney, thyroid, or gastrointestinal disorders, and those with diabetes, hypertension, coronary heart disease, inflammatory disease, polycystic ovary syndrome, rheumatoid arthritis, recent surgery, cancer, and stroke were not included. Additional exclusion criteria were: pregnancy, lactation, smoking, alcohol intake during six months prior to the study, adhering to a specific diet within the last six months, use of medications such as corticosteroids, anticoagulant, antidiuretic, hepatotoxic drugs, antidiabetic drugs, lipidlowering agents, and anti-obesity drugs over three months prior to the study.
Written informed consent was obtained from all participants before enrollment. Subjects underwent inperson interviews to determine medical history.

Dietary Intake and Physical Activity Assessment

A three-day food diary (two weekdays and one weekend day) was used to assess dietary intakes of the subjects. The Nutritionist IV software (First Databank, San Bruno, CA, USA) modified for Iranian foods was applied for dietary data analysis. In this study, twenty-one food groups were included in factor analysis. Data regarding food groups are presented in Table 1.

The level of physical activity of the participants was estimated by international physical activity questionnaireshort form (IPAQ-SF). The validity of this questionnaire has been confirmed previously. ${ }^{25}$ Responses were converted to Metabolic Equivalent Task minutes per week (METminutes/week). The participants were categorized as having high, moderate, or low level of activity, based on the IPAQ scoring protocol.

Assessment of Anthropometric Indices

All anthropometric indices were assessed by a trained nutritionist. Body weight was measured using a scale (Seca, Germany) with a precision of $0.1 \mathrm{~kg}$, in light clothing. Height was measured without shoes using a stadiometer with a precision of $0.1 \mathrm{~cm}$. BMI was calculated as weight (kg) divided by height squared $\left(\mathrm{m}^{2}\right)$. WC was measured at midpoint between the top of the iliac crest and the lower margin of the least palpable rib, by a stretch-resistant tape. Hip circumference (HC) was assessed at the widest part of the hip at the level of the greater trochanter. Body fat mass was assessed by Bioelectrical impedance analysis (BIATanita MC-780, Amsterdam).

\section{Biochemical Assays}

After 12-14 h overnight fast, blood samples were collected and stored at $-70^{\circ} \mathrm{C}$ until biochemical analysis. Serum concentrations of total cholesterol (TC), triglycerides (TG), high-density lipoprotein cholesterol (HDL-C), fasting blood sugar (FBS), ALT, and aspartate aminotransferase (AST) were assessed through enzymatic methods by commercial kits (Pars Azmoon Co., Tehran, Iran). Low-density lipoprotein cholesterol (LDL-C) was measured with the use of the Friedewald formula. The enzyme-linked immunosorbent assay (ELISA) method was used to assess serum insulin level using commercial kits (Monobind, Lake Forest, CA, USA). The homeostatic model (HOMA-IR) was calculated using the following formula:

HOMA-IR $=[$ fasting insulin $(\mu I U / m L) \times F B S(m g / d L)] / 405$

NAFLD Diagnosis

All patients underwent ultrasonography for determining 
Table 1. Food Grouping Used in the Factor Analysis

\begin{tabular}{|c|c|}
\hline Food Groups & Food Items \\
\hline Vegetables & $\begin{array}{l}\text { Lettuce, all kinds of cabbage, cucumber, tomato, spinach, mushroom, garlic, carrot, onions, eggplant, celery, green peas, green } \\
\text { beans, turnip, maize, bell peppers, squash, tomato }\end{array}$ \\
\hline Legumes & Beans, chickpeas, broad beans, lentils, soybeans, lima beans, mung beans \\
\hline Fruits & $\begin{array}{l}\text { Apple, cherries, grapefruit, apricots, plum, kiwi, strawberry, oranges, grapes, berries, dates, barberry, banana, pomegranate, melon, } \\
\text { tangerine, lemon, raisin, mulberry, persimmon, peach, cantaloupe, watermelon, pear, fruit juices }\end{array}$ \\
\hline Low-fat dairy & Yogurt, dough (yogurt drink), milk, cheese \\
\hline White meat & Chicken, turkey, ostrich, fish, seafood \\
\hline Vegetable oils & Vegetable oils \\
\hline Whole grains & Barely bread, whole wheat, wheat germ, oat, bulgur, corn flakes \\
\hline Nuts & Peanut, almond, pistachio, walnut, hazelnut, roasted seeds \\
\hline Refined grains & White bread, rice, flour, macaroni, noodle, biscuit, crackers, cake \\
\hline Sweets and desserts & Jam, honey, candy, sugar, chocolate, pastry \\
\hline Hydrogenated fats & Animal fats, butter, margarine \\
\hline Red and processed meat & Beef, lamb, camel, sausages, hamburger, processed meats, organ meats \\
\hline Soft drinks & Soft drinks \\
\hline High-fat dairy & Whole milk, Chocolate milk, high-fat yogurt, cream cheese, cream, ice cream, pizza cheese, curd \\
\hline Fried potato & French fries, chips \\
\hline Pizza & Pizza \\
\hline Egg & Egg \\
\hline Cooked potato & Cooked potato \\
\hline Pickle & Pickle \\
\hline Tea and coffee & Tea and coffee \\
\hline Salt & Salt \\
\hline
\end{tabular}

fatty liver by a single expert radiologist. Echogenicity grading was performed using SonoAce X4 ultrasound system (South Korea). NAFLD was defined as the presence of moderate-severe hepatic steatosis in the absence of excessive intake of alcoholic beverages.

\section{Statistical Analysis}

All statistical procedures were done using SPSS software (IBM, Armonk, USA). Dietary patterns were determined through factor analysis. The eigenvalues and the scree plot were applied to decide on the number of factors retained. We performed a principal component analysis with varimax rotation in order to make the factors that interpreted the maximum variability of the data. A factor score was created for participants by summing intakes of food groups weighted by their factor loading. The factor scores were further classified into tertiles, where the first tertile represented low adherence and the third tertile displayed high adherence to the dietary pattern. Homogeneity of variance was tested using Leven's test for equality of variances and in all cases, the results showed that the assumption was satisfied for all variables.

The normal probability plot was applied to check the normal distribution of data. Independent-sample $t$-test was applied to compare the means of normally distributed variables. For values with non-normal distribution, the Mann-Whitney U test was used. Normality was confirmed for all variables except for HOMA-IR. The chi-square test was applied to determine the association between two categorical variables. To assess the relationship between food groups and NAFLD, multivariable-adjusted odds ratio (OR) obtained from the logistic regression analysis was performed. The relationship between dietary patterns and NAFLD was also determined from the ORs using the multivariate logistic regression adjusted for potential confounders. We defined two models to compute multivariable-adjusted ORs. The models were as follow: the first model was the crude model. The second model was adjusted for sex, education, physical activity, BMI, and WC. In addition to matching age in the design of the study, we entered it in the models and adjusted the relationships and effect sizes for the residual effects of this variable.

\section{Results}

In this study, two major dietary patterns were extracted. We labeled these factors as follows: the vegetable, legume, fruit, and low-fat dairy product (VLFD) dietary pattern; and the sweet, hydrogenated fat, red and processed meat, and soft drink (SHMS) dietary pattern (Table 2).

As shown in Table 3, NAFLD patients were less physically active compared to controls $(P<0.001)$. Moreover, weight, WC, BMI, body fat mass, serum AST, ALT, TG, and HOMA-IR were significantly higher in NAFLD patients compared to controls $(P<0.05)$.

Participants in the third tertile of the VLFD dietary pattern showed significantly lower weight, WC, BMI, body fat mass, serum levels of ALT and TG, and a lower 
Table 2. Factor Loading Matrix for Major Dietary Patterns

\begin{tabular}{lll}
\hline \multirow{2}{*}{ Food Groups } & \multicolumn{2}{c}{ Dietary Patterns } \\
\cline { 2 - 3 } & VLFD & SHMS \\
\hline Vegetables & 0.733 & \\
\hline Legumes & 0.611 & \\
\hline Fruits & 0.497 & \\
\hline Low-fat dairy & 0.441 & 0.311 \\
\hline White meat & 0.409 & \\
\hline Vegetable oils & 0.402 & \\
\hline Whole grains & 0.391 & \\
\hline Nuts & 0.361 & 0.447 \\
\hline Refined grains & -0.309 & 0.711 \\
\hline Sweets and desserts & & 0.678 \\
\hline Hydrogenated fats & & 0.658 \\
\hline Red and processed meat & & 0.571 \\
\hline Soft drinks & & 0.532 \\
\hline High-fat dairy & & 0.471 \\
\hline Fried potato & & 0.425 \\
\hline Pizza & & 0.382 \\
\hline Egg & & 0.374 \\
\hline Cooked potato & & 0.325 \\
\hline Pickle & & \\
\hline Tea and coffee & & \\
\hline Salt & & \\
\hline Variance explained & & \\
\hline LF vera & \\
\hline
\end{tabular}

VLFD, vegetables, legumes, fruits, and low-fat dairy; SHMS, sweets, hydrogenated fats, red processed meat, and soft drinks.

Absolute values $<0.3$ were not displayed for simplicity.

consumption of energy, carbohydrate, and fat, but a higher protein intake and a greater serum level of HDL-C compared with those in the first tertile. However, there were no significant difference in physical activity level, serum levels of AST, TC, LDL-C, FBS, and HOMA-IR across tertiles of this pattern. Moreover, subjects in the third tertiles of the SHMS dietary exhibited higher weight, WC, BMI, body fat mass, serum levels of ALT and TG, and a greater intake of energy, carbohydrate, and fat, but a lower protein intake compared with those in the lowest tertile (Table 4).

Participants in the third tertile of red or processed meat consumption (OR: 2.68; 95\% CI: 1.31-4.16), hydrogenated fats (OR: 2.66; 95\% CI: 1.42-4.70), sweets and desserts (OR: 2.59; 95\% CI: 0.89-3.48), and soft drinks (OR: 2.61; 95\% CI: 1.32-5.22), showed a greater risk of NAFLD than those in the first tertile. Conversely, patients in the highest tertile of vegetable consumption exhibited a lower risk of NAFLD (OR: 0.34; 95\% CI: 0.16-0.81) compared with those in the lowest tertile (Table 5).

As shown in Table 6, the VLFD dietary pattern was significantly associated with lower odds of NAFLD. In the first model, high adherence to the VLFD dietary pattern was related to lower odds of NAFLD compared to low adherence. Following further adjustment based on model 2 , a significant downward trend was observed in the odds
Table 3. Characteristics of NAFLD Cases and Controls

\begin{tabular}{|c|c|c|c|}
\hline & $\begin{array}{c}\text { Cases } \\
(n=105)\end{array}$ & $\begin{array}{l}\text { Controls } \\
(n=105)\end{array}$ & $P$ \\
\hline Age (years) & $45.6 \pm 9.1$ & $45.4 \pm 9.3$ & $0.74^{\mathrm{a}}$ \\
\hline Male (n\%) & 42.8 & 42.8 & $0.50^{\mathrm{b}}$ \\
\hline Physical activity* (\%) & & & $0.001^{\mathrm{b}}$ \\
\hline Low & $64(60.9)$ & $45(42.8)$ & \\
\hline Moderate & $35(33.3)$ & $49(46.6)$ & \\
\hline High & $6(5.8)$ & $11(10.4)$ & \\
\hline Weight (kg) & $87 \pm 17.5$ & $79.1 \pm 14.5$ & $0.01^{\mathrm{a}}$ \\
\hline Waist circumference $(\mathrm{cm})$ & $102.3 \pm 13.5$ & $96.3 \pm 15.9$ & $<0.001^{\text {a }}$ \\
\hline $\mathrm{BMI}\left(\mathrm{kg} / \mathrm{m}^{2}\right)$ & $33.8 \pm 7.7$ & $30.3 \pm 6.5$ & $<0.001^{\text {a }}$ \\
\hline Body fat mass (kg) & $31.1 \pm 5.1$ & $27.30 \pm 6.9$ & $<0.01^{\mathrm{a}}$ \\
\hline $\mathrm{AST}(\mathrm{U} / \mathrm{L})$ & $24.9 \pm 8.1$ & $20.3 \pm 7.6$ & $<0.001^{a}$ \\
\hline $\mathrm{ALT}(\mathrm{U} / \mathrm{L})$ & $31.7 \pm 13.1$ & $21.4 \pm 9.3$ & $<0.001^{a}$ \\
\hline $\mathrm{TC}(\mathrm{mg} / \mathrm{dL})$ & $189 \pm 21.9$ & $181.7 \pm 24.8$ & $0.12^{\mathrm{a}}$ \\
\hline $\mathrm{TG}(\mathrm{mg} / \mathrm{dL})$ & $132.8 \pm 31.8$ & $107.3 \pm 39.1$ & $<0.01^{\mathrm{a}}$ \\
\hline LDL-C (mg/dL) & $124.7 \pm 28.9$ & $119.6 \pm 36.2$ & $0.33^{a}$ \\
\hline $\mathrm{HDL}-\mathrm{C}(\mathrm{mg} / \mathrm{dL})$ & $38.37 \pm 14.6$ & $40.5 \pm 13.9$ & $0.06^{\mathrm{a}}$ \\
\hline FBS (mg/dL) & $94.3 \pm 10.7$ & $91.2 \pm 13.7$ & $0.23^{\mathrm{a}}$ \\
\hline HOMA-IR & $3.9(1.1,6.3)^{\mathrm{d}}$ & $2.2(1.4-6.8)^{d}$ & $<0.01^{\mathrm{c}}$ \\
\hline
\end{tabular}

NAFLD, Non-alcoholic fatty liver disease; BMI, Body mass index; MET-h, Metabolic equivalent hours; AST, Aspartate aminotransferase; ALT, Alanine aminotransferase; TC, Total cholesterol; TG, Triglyceride; LDL-C, Low-density lipoprotein cholesterol; HDL-C, High-density lipoprotein cholesterol; FBS, Fasting blood sugar; HOMA-IR, Homeostasis model assessment of insulin resistance.

Data are reported as mean \pm SD or percentage as appropriate.

${ }^{a}$ Independent samples t-test using equal variances (assessed and satisfied through Leven's test).

${ }^{b}$ Chi-squared test.

${ }^{\mathrm{c}}$ Mann-Whitney $U$ test.

${ }^{\mathrm{d}}$ Data were expressed as Median (25th and 75th percentiles)

*<600 MET/h per week: "low", 600-3000 MET/h per week: "moderate", >3000 MET/h per week: "high".

of NAFLD with increasing the adherence to this pattern. In contrast, adherence to the SHMS dietary pattern was significantly related to the increased risk of NAFLD. A significant increase was also observed in the odds of NAFLD with increased adherence to this pattern after adjustment for cofounders based on model 2 (Table 6).

\section{Discussion}

Dietary patterns identified by factor analysis provide a more realistic representation of daily eating habits. ${ }^{26,27}$ Based on our extensive search, this is the first study to assess the relationship between dietary patterns and NAFLD incidence among Iranian adults. In the current study, two major dietary patterns were extracted including VLFD and SMHS dietary patterns. The results demonstrated that the VLFD and SMHS dietary patterns were associated with NAFLD inversely and directly, respectively. Additionally, the VLFD dietary pattern was inversely associated with weight, WC, BMI, body fat mass, serum levels of ALT and TG, and intakes of energy, carbohydrate, and fat. However, the SHMS pattern was indicated to be positively associated with weight, WC, BMI, body fat mass, serum levels of ALT and TG, and intakes of energy, carbohydrate, and fat. Besides, among the major food groups, vegetable 
Table 4. Characteristics of the Study Participants Across Tertiles (T) of the Dietary Pattern Scores

\begin{tabular}{|c|c|c|c|c|c|c|}
\hline \multirow{2}{*}{ Variables } & \multicolumn{3}{|c|}{ VLFD Dietary Pattern } & \multicolumn{3}{|c|}{ SHMS Dietary Pattern } \\
\hline & T1 (Lowest) $(n=70)$ & T3 (Highest) $(\mathrm{n}=70)$ & $P$ & T1 (Lowest) $(\mathrm{n}=70)$ & T3 (Highest) $(\mathrm{n}=70)$ & $P$ \\
\hline Age (years) & $49.7 \pm 5.9$ & $48.3 \pm 4.1$ & 0.43 & $48.1 \pm 4.8$ & $46.3 \pm 3.3$ & 0.09 \\
\hline Physical activity (\%) & & & 0.08 & & & 0.1 \\
\hline Low & $26(37.1)$ & $25(35.7)$ & & $28(40.1)$ & $29(41.4)$ & \\
\hline Moderate & $33(47.1)$ & $34(48.5)$ & & $30(42.8)$ & $31(44.3)$ & \\
\hline High & $10(14.2)$ & $11(15.7)$ & & $12(17.1)$ & $10(14.3)$ & \\
\hline Weight (kg) & $81.4 \pm 6.7$ & $77.2 \pm 4.3$ & 0.001 & $78.7 \pm 11.2$ & $83.3 \pm 9.9$ & 0.001 \\
\hline Waist circumference $(\mathrm{cm})$ & $94.2 \pm 10.7$ & $87.2 \pm 9.4$ & 0.006 & $88.9 \pm 10.8$ & $95.6 \pm 12.1$ & $<0.001$ \\
\hline $\mathrm{BMI}\left(\mathrm{kg} / \mathrm{m}^{2}\right)$ & $29.7 \pm 4.7$ & $27.9 \pm 5.3$ & 0.001 & $26.1 \pm 5.1$ & $28.9 \pm 6.9$ & $<0.001$ \\
\hline Body fat mass (kg) & $31.1 \pm 6.2$ & $30.3 \pm 8.3$ & 0.03 & $28.3 \pm 7.9$ & $30.6 \pm 8.8$ & 0.003 \\
\hline Energy intake (kcal/d) & $2245.1 \pm 321$ & $1987.8 \pm 183$ & $<0.001$ & $1954.3 \pm 211$ & $2311.9 \pm 196$ & $<0.001$ \\
\hline Carbohydrate intake (g/d) & $291 \pm 18.8$ & $276 \pm 23.9$ & 0.002 & $265.6 \pm 33.5$ & $284.2 \pm 41.4$ & 0.003 \\
\hline Fat intake $(\mathrm{g} / \mathrm{d})$ & $68.4 \pm 18.4$ & $61.3 \pm 13.9$ & 0.001 & $58.8 \pm 11.7$ & $67.7 \pm 12.3$ & 0.001 \\
\hline Protein intake (g/d) & $61.7 \pm 11.1$ & $64.5 \pm 9.7$ & 0.007 & $67.4 \pm 18.3$ & $59.7 \pm 14.7$ & 0.001 \\
\hline AST (U/L) & $23.5 \pm 6.9$ & $23.9 \pm 7.2$ & 0.27 & $24.3 \pm 7.7$ & $25.4 \pm 9.4$ & 0.43 \\
\hline ALT (U/L) & $27.4 \pm 9.8$ & $25.1 \pm 10.4$ & 0.02 & $24.4 \pm 8.1$ & $27.3 \pm 6.6$ & 0.02 \\
\hline TC (mg/dL) & $189.4 \pm 18.4$ & $191 \pm 23.9$ & 0.61 & $194.6 \pm 34.6$ & $201.6 \pm 31.3$ & 0.14 \\
\hline TG (mg/dL) & $112.8 \pm 21.8$ & $87 \pm 11.7$ & $<0.001$ & $91.6 \pm 14.2$ & $104.9 \pm 21.9$ & 0.001 \\
\hline LDL-C (mg/dL) & $129.5 \pm 28.8$ & $126 \pm 32.2$ & 0.54 & $131 \pm 34.2$ & $133.5 \pm 38.1$ & 0.16 \\
\hline $\mathrm{HDL}-\mathrm{C}(\mathrm{mg} / \mathrm{dL})$ & $42.4 \pm 11.1$ & $45.4 \pm 13.6$ & 0.003 & $46.3 \pm 12.9$ & $45.9 \pm 10.8$ & 0.29 \\
\hline FBS (mg/dL) & $92.9 \pm 13.6$ & $91.7 \pm 17.6$ & 0.13 & $92.7 \pm 11.8$ & $94.7 \pm 17.9$ & 0.14 \\
\hline HOMA-IR & $2.2(1.1,6.3)$ & $2.04(1.2,5.8)$ & 0.11 & $2.1(1.1,6.1)$ & $2.3(1.01,6.4)$ & 0.63 \\
\hline
\end{tabular}

BMI, Body mass index; AST, Aspartate aminotransferase; ALT, Alanine aminotransferase; TC, Total cholesterol; TG, Triglyceride; LDL-C, Low-density lipoprotein cholesterol; HDL-C, High-density lipoprotein cholesterol; FBS, Fasting blood sugar; HOMA-IR, Homeostasis model assessment of insulin resistance. VLFD, vegetables, legumes, fruits, and low-fat dairy; SHMS, sweets, hydrogenated fats, red processed meat, and soft drinks.

Mean \pm SD are presented for normally distrusted data; Median (25th and 75th percentiles) are presented for data not normally distributed (HOMA-IR)

Independent samples t-test using equal variances (assessed and satisfied through Levene's test).

Table 5. Odds Ratios $(95 \% \mathrm{Cl})$ for NAFLD Across Tertiles (T) of Food Groups

\begin{tabular}{|c|c|c|c|c|}
\hline Food Groups & T1 (Low Adherence) & T2 & T3 (High Adherence) & $P$-trend \\
\hline Vegetables & Ref & $0.73(0.31-1.29)$ & $0.34(0.16-0.81)$ & 0.01 \\
\hline Legumes & Ref & $0.68(0.21-1.32)$ & $0.74(0.61-1.67)$ & 0.24 \\
\hline Fruits & Ref & $0.63(0.16-1.71)$ & $0.54(0.19-1.56)$ & 0.52 \\
\hline Low-fat dairy & Ref & $0.51(0.14-1.51)$ & $0.46(0.16-1.32)$ & 0.14 \\
\hline White meat & Ref & $1.36(0.64-3.94)$ & $1.11(0.48-4.17)$ & 0.63 \\
\hline Vegetable oils & Ref & $1.05(0.38-1.87)$ & $0.74(0.29-1.44)$ & 0.52 \\
\hline Whole grains & Ref & $0.61(0.38-1.44)$ & $0.53(0.31-1.21)$ & 0.21 \\
\hline Nuts & Ref & $0.93(0.72-1.48)$ & $0.84(0.61-1.09)$ & 0.08 \\
\hline Refined grains & Ref & $1.33(0.69-2.65)$ & $2.11(0.72-4.37)$ & 0.32 \\
\hline Sweets and desserts & Ref & $1.32(0.72-2.41)$ & $2.59(1.11-3.48)$ & 0.01 \\
\hline Hydrogenated fats & Ref & $1.74(1.24-3.11)$ & $2.66(1.42-4.70)$ & 0.02 \\
\hline Red and processed meat & Ref & $1.34(0.89-2.91)$ & $2.68(1.31-4.16)$ & $<0.01$ \\
\hline Soft drinks & Ref & $1.31(0.63-2.45)$ & $2.61(1.32-5.22)$ & $<0.01$ \\
\hline High-fat dairy & Ref & $1.21(0.64-1.41)$ & $2.01(0.81-3.11)$ & 0.28 \\
\hline Fried potato & Ref & $1.01(0.84-1.69)$ & $1.44(0.91-2.78)$ & 0.46 \\
\hline Pizza & Ref & $1.29(0.71-2.79)$ & $2.22(0.88-3.45)$ & 0.41 \\
\hline Egg & Ref & $1.18(0.51-2.31)$ & $1.38(0.71-2.59)$ & 0.54 \\
\hline Cooked potato & Ref & $1.19(0.79-2.34)$ & $2.04(0.86-3.26)$ & 0.59 \\
\hline Pickle & Ref & $1.11(0.71-1.41)$ & $2.01(0.84-2.61)$ & 0.61 \\
\hline Tea and coffee & Ref & $1.09(0.56-1.33)$ & $1.52(0.61-2.44)$ & 0.66 \\
\hline Salt & Ref & $1.29(0.72-1.55)$ & $2.31(0.88-3.59)$ & 0.22 \\
\hline
\end{tabular}

NAFLD, Non-alcoholic fatty liver disease. 
Table 6. Odds ratios (95\% Cl) for NAFLD Across Tertiles (T) of Dietary Pattern Scores

\begin{tabular}{|c|c|c|c|c|}
\hline Dietary Pattern & T1 (Low Adherence) & T2 & T3 (High Adherence) & $P$-trend \\
\hline \multicolumn{5}{|l|}{ VLFL $(n=210)$} \\
\hline \multicolumn{5}{|l|}{ Model $1^{\text {a }}$} \\
\hline \multicolumn{5}{|l|}{ Age (years) } \\
\hline $30-39$ & Ref & $0.52(0.27-1.27)$ & $0.31(0.18-0.95)$ & 0.001 \\
\hline $40-49$ & Ref & $0.61(0.33-1.33)$ & $0.41(0.11-0.83)$ & 0.006 \\
\hline $50-59$ & Ref & $0.71 \quad(0.21-1.27)$ & $0.66(0.16-0.94)$ & 0.032 \\
\hline \multicolumn{5}{|l|}{ Model $2^{b}$} \\
\hline \multicolumn{5}{|l|}{ Age (years) } \\
\hline $30-39$ & Ref & $0.39(0.17-1.41)$ & $0.27(0.12-0.79)$ & 0.012 \\
\hline $40-49$ & Ref & $0.44(0.24-1.27)$ & $0.33(0.11-0.84)$ & 0.025 \\
\hline $50-59$ & Ref & $0.55(0.31-1.22)$ & $0.28(0.16-0.93)$ & 0.033 \\
\hline \multicolumn{5}{|l|}{ SHMS $(n=210)$} \\
\hline \multicolumn{5}{|l|}{ Model $1^{\text {a }}$} \\
\hline \multicolumn{5}{|l|}{ Age (year) } \\
\hline $30-39$ & Ref & $1.53(0.55-3.01)$ & $2.79(1.21-3.87)$ & 0.041 \\
\hline $40-49$ & Ref & $1.64(0.74-3.44)$ & $2.73(1.31-5.83)$ & 0.033 \\
\hline $50-59$ & Ref & $2.01(1.05-4.42)$ & $4.44(1.81-7.12)$ & 0.039 \\
\hline \multicolumn{5}{|l|}{ Model $2^{\mathrm{b}}$} \\
\hline \multicolumn{5}{|l|}{ Age (year) } \\
\hline $30-39$ & Ref & $1.69(0.69-3.79)$ & $2.93(1.54-5.12)$ & 0.026 \\
\hline $40-49$ & Ref & $1.21(0.65-3.45)$ & $3.17(1.04-6.66)$ & 0.033 \\
\hline $50-59$ & Ref & $1.71(0.77-4.44)$ & $4.22(1.22-6.91)$ & 0.013 \\
\hline
\end{tabular}

NAFLD, Non-alcoholic fatty liver disease; VLFD, vegetables, legumes, fruits, and low-fat dairy; SHMS, sweets, hydrogenated fats, red processed meat, and soft drinks.

Multivariable-adjusted odds ratio (OR) obtained from the logistic regression analysis.

${ }^{\text {a }}$ Crude model (model 1).

${ }^{\mathrm{b}}$ Adjusted for sex, education, physical activity, BMI, and waist circumference (model 2).

consumption was found to play a protective role against NAFLD.

The results obtained in this study are in line with those of some previous studies. In this context, a case-control study on the elderly Brazilian population reported that NAFLD is inversely associated with adherence to the healthy dietary pattern and is also related to the regional snacks pattern directly, which is characterized by the presence of regional foods with high carbohydrates and high glycemic load, after adjusting for confounding variables. ${ }^{19}$ Similarly, in another study, high-carbohydrate/sweet pattern scores were positively related to the prevalence of NAFLD in female subjects. ${ }^{28}$ In a study on 999 Chinese adults, after adjusting for potential confounders, "animal food" and "grains-vegetables" dietary patterns were found to be directly and inversely associated with the prevalence of NAFLD, respectively. ${ }^{29}$ In a Greek case-control study, subjects in the 4th quartile of the "fast-food type" dietary pattern showed 3.9 times higher odds for NAFLD compared to the individuals in the 1 st quartile, after adjustment for confounders. ${ }^{30}$ Similarly, a high meat/fast food pattern significantly increased the risk of NAFLD in Lebanese patients, whereas the "traditional Lebanese" pattern which is characterized by high consumption of vegetables, legumes, and vegetable oil/olives was inversely related to the odds of NAFLD. ${ }^{31}$ Moreover, a Western dietary pattern was related to the increased risk of NAFLD in a population-based cohort of adolescents. ${ }^{14}$ However, no association was found between the Western and high-carbohydrate pattern and NAFLD risk in Korean adults. ${ }^{27}$ Dietary patterns change among ethnicities, cultural groups, gender, and they may also vary as a result of personal preferences and food availability, which may explain the discrepancy in the obtained results. ${ }^{32,33}$

The Mediterranean diet, which is rich in fibers, antioxidants, and monounsaturated fats, has been demonstrated to be protective against NAFLD. ${ }^{34,35}$ In this regard, Kontogianni et $\mathrm{al}^{36}$ investigated the effect of adherence to the Mediterranean diet on the presence and severity of NAFLD in adult patients. Their findings demonstrated that higher adherence to the Mediterranean diet was associated with lower degrees of insulin resistance (IR) as well as less severe liver disease in patients with NAFLD. In the present study, the "VLFD" pattern had the major components of the Mediterranean diet containing a wide range of nutraceuticals such as phytosterols and polyphenols, which can attenuate hepatic steatosis through antioxidant and anti-inflammatory activities. Polyphenols may have hepatoprotective effects by increasing fatty acid oxidation and modulation of IR, oxidative stress, and inflammation, which are known as the major factors involved in the pathogenesis of NAFLD. ${ }^{37}$

The "SHMS" dietary pattern includes most of the main components of the Western diet. Also, it has been reported that an inappropriate dietary pattern like the Western food style, which is characterized by high consumption of soft 
drinks, saturated fats, trans fats, red and processed meat, and refined grains, may contribute to hepatic steatosis risk through increased total and visceral fat mass, IR, and increased hepatic de novo lipogenesis. ${ }^{4,14,38,39}$ In this study, although the consumption of tea and coffee was not associated with odds for NAFLD significantly, coffee and tea were included in the SMHS dietary pattern. In contrast to our findings, some previous studies have reported that consumption of tea and coffee can have protective effects against NAFLD. ${ }^{18,40}$ The discrepancy between these results might be due to the consumption of sugar and sweets along with tea or coffee. In the current study, the associations of VLFD and SMHS dietary patterns with NAFLD remained to be significant even after controlling for confounding variables, which indicated that these dietary patterns directly act on NAFLD.

The most important strength of the current research was that this is the first study investigating the association between dietary patterns and NAFLD in patients newly diagnosed with NAFLD, receiving no treatment. However, a few limitations of this study are also notable. Firstly, some inflated OR estimates and confidence limits may be an indication of sparse-data bias. ${ }^{41,42}$ Finally, due to the case-control design of this study, the causality effect could not be indicated; therefore, our findings need to be confirmed in future prospective studies.

In conclusion, the present study indicated that the VLFD dietary pattern was associated with reduced likelihood of having NAFLD, while the SHMS dietary pattern was related to higher likelihood. Thus, the VLFD dietary pattern might be useful in the nutritional strategies for NAFLD patients. However, further studies with larger sample sizes and prospective design are warranted to confirm our findings.

\section{Authors' Contribution}

HT drafted the manuscript and analyzed and interpreted the data; MAJ was the statistic counsellor; AO and MSA contributed to the final revision of the manuscript. All authors approved the final version of this manuscript.

\section{Conflict of Interest Disclosures}

All authors declare that there is no conflict of interest.

\section{Ethical Statement}

The Ethical Committee of Tabriz University of Medical Sciences approved the study protocol.

\section{Funding}

The study was financially supported by the Nutrition Research Center of Tabriz University of Medical Sciences, and Iran National Science Foundation (INSF).

\section{Acknowledgements}

We sincerely thank the patients who participated in the present study.

\section{References}

1. Younossi ZM, Marchesini G, Pinto-Cortez H, Petta S. Epidemiology of Nonalcoholic Fatty Liver Disease and Nonalcoholic Steatohepatitis: Implications for Liver
Transplantation. Transplantation. 2019;103(1):22-7. doi: 10.1097/tp.0000000000002484

2. Fabbrini E, Sullivan S, Klein S. Obesity and nonalcoholic fatty liver disease: biochemical, metabolic, and clinical implications. Hepatology. 2010;51(2):679-89. doi:10.1002/ hep. 23280

3. Perdomo CM, Frühbeck G, Escalada J. Impact of Nutritional Changes on Nonalcoholic Fatty Liver Disease. Nutrients. 2019;11(3):677. doi: 10.3390/nu11030677.

4. Tutunchi H, Saghafi-Asl M, Asghari-Jafarabadi M, Ostadrahimi A. The relationship between severity of liver steatosis and metabolic parameters in a sample of Iranian adults. BMC Res Notes. 2020;13:218. doi: 10.1186/s13104-020-05059-5.

5. Ashtari S, Pourhoseingholi MA, Zali MR. Non-alcohol fatty liver disease in Asia: Prevention and planning. World J Hepatol. 2015;7(13):1788-96. doi: 10.4254/wjh.v7.i13.1788.

6. Tutunchi H, Asghari Jafarabadi M, Hoojeghani S, Tabrizi S, Farrin N, Ostadrahimi A. General and abdominal obesity is related to socioeconomic status and food choices: a crosssectional study. Nutr Food Sci. 2019;50:61-73. doi:10.1108/ NFS-02-2019-0056

7. Zou TT, Zhang C, Zhou YF, Han YJ, Xiong JJ, Wu XX, et al. Lifestyle interventions for patients with nonalcoholic fatty liver disease: a network meta-analysis. Eur J Gastroenterol Hepatol. 2018;30(7):747-55. doi: 10.1097/MEG.0000000000001135.

8. Riazi K, Raman M, Taylor L, Swain MG, Shaheen AA. Dietary patterns and components in nonalcoholic fatty liver disease (NAFLD): what key messages can health care providers offer? Nutrients. 2019;11(12):2878. doi: 10.3390/nu11122878.

9. Tutunchi H, Ostadrahimi A, Hosseinzadeh-Attar MJ, Miryan M, Mobasseri M, Ebrahimi-Mameghani M. A systematic review of the association of neuregulin 4, a brown fat-enriched secreted factor, with obesity and related metabolic disturbances. Obes Rev. 2019; 21(2):e12952. doi:10.1111/obr.12952.

10. Ullah R, Rauf N, Nabi G, Ullah H, Shen Y, Zhou YD, et al. Role of nutrition in the pathogenesis and prevention of nonalcoholic fatty liver disease: Recent Updates. Int J Biol Sci. 2019;15(2):265-76. doi: 10.7150/ijbs.30121.

11. Katsagoni CN, Georgoulis M, Papatheodoridis GV, Fragopoulou E, loannidou P, Papageorgiou M, et al. Associations between lifestyle characteristics and the presence of nonalcoholic fatty liver disease: a case-control study. Metab Syndr Relat Disord. 2017;15(2):72-9. doi: 10.1089/met.2016.0105.

12. Tutunchi H, Saghafi-Asl M, Ostadrahimi A. A systematic review of the effects of oleoylethanolamide, a high affinity endogenous ligand of PPAR-alpha, on the management and prevention of obesity. Clin Exp Pharmacol Physiol. 22020; 47 (4):543-552. doi: 10.1111/1440-1681.13238.

13. Mirmiran P, Amirhamidi Z, Ejtahed HS, Bahadoran Z, Azizi F. Relationship between Diet and Non-alcoholic Fatty Liver Disease: A Review Article. Iran J Public Health. 2017;46(8):1007-17.

14. Oddy $\mathrm{WH}$, Herbison $\mathrm{CE}$, Jacoby $\mathrm{P}$, Ambrosini GL, O'Sullivan TA, Ayonrinde OT, et al. The Western dietary pattern is prospectively associated with nonalcoholic fatty liver disease in adolescence. Am J Gastroenterol. 2013;108(5):778-85. doi: 10.1038/ajg.2013.95.

15. Fan JG, Cao HX. Role of diet and nutritional management in non-alcoholic fatty liver disease. J Gastroenterol Hepatol. 2013;28 Suppl 4:81-7. doi:10.1111/jgh.12244.

16. Moeller SM, Reedy J, Millen AE, Dixon LB, Newby PK, Tucker $\mathrm{KL}$, et al. Dietary patterns: challenges and opportunities in dietary patterns research an Experimental Biology workshop, April 1, 2006. J Am Diet Assoc. 2007;107(7):1233-9. doi: 10.1016/j.jada.2007.03.014.

17. Jacques PF, Tucker KL. Are dietary patterns useful for understanding the role of diet in chronic disease? Am J Clin Nutr. 2001;73(1):1-2. doi: 10.1093/ajcn/73.1.1. 
18. Soleimani D, Ranjbar G, Rezvani R, Goshayeshi L, Razmpour F, Nematy M. Dietary patterns in relation to hepatic fibrosis among patients with nonalcoholic fatty liver disease. Diabetes Metab Syndr Obes. 2019;12:315-24. doi: 10.2147/DMSO. S198744.

19. Adriano LS, Sampaio HA, Arruda SP, Portela CL, de Melo MLP, Carioca AA, et al. Healthy dietary pattern is inversely associated with non-alcoholic fatty liver disease in elderly. $\mathrm{Br} \mathrm{J}$ Nutr. 2016;115(12):2189-95. doi: 10.1017/S0007114516001410.

20. Georgoulis M, Kontogianni MD, Margariti A, Tiniakos D, Fragopoulou E, Zafiropoulou R, et al. Associations between dietary intake and the presence of the metabolic syndrome in patients with non-alcoholic fatty liver disease. J Hum Nutr Diet. 2015;28(4):409-15. doi: 10.1111/jhn.12323.

21. Zelber-Sagi S, Salomone F, Mlynarsky L. The Mediterranean dietary pattern as the diet of choice for non-alcoholic fatty liver disease: Evidence and plausible mechanisms. Liver Int 2017;37(7):936-49. doi: 10.1111/liv.13435.

22. Abenavoli L, Di Renzo L, Boccuto L, Alwardat N, Gratteri S, De LorenzoA. Health benefits of Mediterranean diet in nonalcoholic fatty liver disease. Expert Rev Gastroenterol Hepatol. 2018;12(9):873-81. doi: 10.1080/17474124.2018.1503947.

23. PASS 15 Power Analysis and Sample Size Software (2017). Kaysville, Utah, USA: NCSS; 2017.

24. Formulae Chow SC, Shao J, Wang H. Sample Size Calculations in Clinical Research. 2nd Ed. Boca Raton: Chapman \& Hall/ CRC; 2008.

25. Craig $\mathrm{CL}$, Marshall $\mathrm{AL}$, Sjostrom $\mathrm{M}$, Bauman $\mathrm{AE}$, Booth $\mathrm{ML}$, Ainsworth $\mathrm{BE}$, et al. International physical activity questionnaire: 12-country reliability and validity. Med Sci Sports Exerc. 2003;35(8):1381-95. doi: 10.1249/01. MSS.0000078924.61453.FB.

26. Esmaillzadeh A, Kimiagar M, Mehrabi $Y$, Azadbakht L, Hu FB, Willett WC. Dietary patterns, insulin resistance, and prevalence of the metabolic syndrome in women. Am J Clin Nutr. 2007;85(3):910-8. doi: 10.1093/ajcn/85.3.910.

27. Chung GE, Youn J, Kim YS, Lee JE, Yang SY, Lim JH, et al. Dietary patterns are associated with the prevalence of nonalcoholic fatty liver disease in Korean adults. Nutrition. 2019;62:32-8. doi: 10.1016/j.nut.2018.11.021.

28. Jia Q, Xia Y, Zhang Q, Wu H, Du H, Liu L, et al. Dietary patterns are associated with prevalence of fatty liver disease in adults. Eur J Clin Nutr. 2015;69(8):914-21.

29. Yang CQ, Shu L, Wang S, Wang JJ, Zhou Y, Xuan YJ, et al. Dietary Patterns Modulate the Risk of Non-Alcoholic Fatty Liver Disease in Chinese Adults. Nutrients. 2015;7(6):4778-91. doi: $10.3390 /$ nu7064778.

30. Kalafati IP, Borsa D, Dimitriou M, Revenas K, Kokkinos A, Dedoussis GV. Dietary patterns and non-alcoholic fatty liver disease in a Greek case-control study. Nutrition. 2019;61:10510. doi: 10.1016/j.nut.2018.10.032.

31. Fakhoury-Sayegh N, Younes H, Heraoui G, Sayegh R. Nutritional profile and dietary patterns of lebanese nonalcoholic fatty liver disease patients: a case-control study. Nutrients. 2017;9(11):1245. doi: 10.3390/nu9111245.

32. Fung TT, Rimm EB, Spiegelman D, Rifai N, Tofler GH, Willett WC, et al. Association between dietary patterns and plasma biomarkers of obesity and cardiovascular disease risk. Am J Clin Nutr. 2001;73(1):61-7. doi: 10.1093/ajcn/73.1.61.

33. Mokhtari Z, Poustchi H, Eslamparast T, Hekmatdoost A. Egg consumption and risk of non-alcoholic fatty liver disease. World J Hepatol. 2017;9(10):503-9. doi: 10.4254/wjh. v9.i10.503.

34. Aller R, Izaola O, de la Fuente B, De Luis Roman DA. Mediterranean diet is associated with liver histology in patients with non alcoholic fatty liver disease. Nutr Hosp. 2015;32(6):2518-24. doi: 10.3305/nh.2015.32.6.10074.

35. Abenavoli L, Boccuto L, Federico A, Dallio M, Loguercio C, Di Renzo L, et al. Diet and Non-Alcoholic Fatty Liver Disease: The Mediterranean Way. Int J Environ Res Public Health. 2019;16(17):3011. doi: 10.3390/ijerph16173011.

36. Kontogianni MD, Tileli N, Margariti A, Georgoulis M, Deutsch $M$, Tiniakos $D$, et al. Adherence to the Mediterranean diet is associated with the severity of non-alcoholic fatty liver disease. Clin Nutr. 2014;33(4):678-83. doi: 10.1016/j. clnu.2013.08.014.

37. Abenavoli L, Milic N, Luzza F, Boccuto L, De Lorenzo A. Polyphenols Treatment in Patients with Nonalcoholic Fatty Liver Disease. J Transl Int Med. 2017;5(3):144-7. doi: 10.1515/ jtim-2017-0027.

38. Yasutake K, Kohjima M, Kotoh K, Nakashima M, Nakamuta M, Enjoji M. Dietary habits and behaviors associated with nonalcoholic fatty liver disease. World J Gastroenterol. 2014;20(7):1756-67. doi: 10.3748/wjg.v20.i7.1756.

39. Schattenberg JM, Bergheim I. Nutritional Intake and the Risk for Non-Alcoholic Fatty Liver Disease (NAFLD). Nutrients. 2019;11(3):588. doi: 10.3390/books978-3-03897-599-1.

40. Petta S, Marchesini G. Coffee and tea breaks for liver health. J Hepatol. 2017;67(2):221-3. doi: 10.1016/j.jhep.2017.04.014

41. Agressti A. Categorical Data Analysis. 3rd ed. Wiley; 2012.

42. Greenland S, Mansournia MA, Altman DG. Sparse-data bias: A problem hiding in plain sight. BMJ. 2016;353: i1981. 